\title{
ALGUNOS CONCEPTOS PARA EL ESTUDIO DE LA INDUCCIÓN SEMÁNTICA DE LA ACCIÓN SOCIAL
}

\section{A FEW CONCEPTS FOR THE STUDY OF SEMANTIC INDUCTION OF SOCIAL ACTION}

\author{
Maynor Antonio Mora Alvarado \\ Universidad Nacional de Costa Rica \\ mmoraa90@hotmail.com
}

Recibido: 14/03/2016 - Aprobado: 16/09/2017

\begin{abstract}
Resumen
En este breve artículo se propone la necesidad de problematizar la teoría de la acción social desde un punto de vista complejo, lo que implica la posibilidad de ampliar las tesis del individualismo metodológico, así como las tesis del sujeto (sea desde un punto de vista liberal o marxista). Esto con el fin de realizar una descripción o acercamiento a la amplitud en cuanto al alcance actual de la acción social y sus derroteros evidentemente sistémicos, por encima de los procesos de constitución y desarrollo de la denominada vida humana consciente.
\end{abstract}

Palabras clave: acción social, actancia, consciencia, sistema, vida

\section{Summary}

In this brief article we propose the need to problematize the theory of social action from a complex point of view, which implies the possibility of amplifying the thesis of methodological individualism, as well as the theses of the subject (be it from a liberal or a Marxist point of view). The end is to carry out a description or approach at the same time, to the range of current reach of social action and its evidently systemic course, over the constitution and development processes of namely conscious human life.

Key words: Social action, Actancy, Consciousness, System, Life.

\section{Introducción}

El presente texto tiene como objetivo la descripción preliminar de algunos conceptos implicados en la explicación de las formaciones sociales 
modernas, a partir, a su vez, de los conceptos de sistema y vida ("mundo de la vida”, según Habermas, 2007a y 2007b; ver, igualmente, Blumenberg, 2013).

En este sentido, se realiza un rápido diagnóstico de las características principales de las formaciones sociales modernas; para luego problematizar los principales conceptos de una perspectiva metateórica relacionada con las causas de tal contexto. El documento se completa con un breve glosario de los principales términos "difíciles" o poco claros en su definiendum preliminar y algunos neologismos utilizados (en lo posible, no más de los estrictamente necesarios), que requerirán posteriores ingresos y un tratamiento mucho más detallado, dentro de una teoría general de la acción social dentro de la modernidad (aunque no exclusiva de la misma), en seguimiento de los aportes de Habermas y Luhmann.

La tesis principal a defender es que el sistema ha colonizado la vida más allá de los márgenes propuestos por Habermas en su Teoría de la acción comunicativa (2007a y 2007b). Y que el sistema se ha decantado, por la ampliación del número de actancias sociales, por un lado, y, por otro, por el control sobre los agentes (actancias con un alto grado de alcance cognitivo), entre ellos el sujeto, mediante productos culturales de naturaleza comunicativa no consciente. De ahí que sea imposible una teoría social exclusivamente pensada desde el punto de vista de la consciencia o de "lo humano" en sentido bio o no biocognitivo (Díaz, 2008; Serres, 2011; Sanfélix, 2003). Lo que indica la necesidad de revitalizar el estudio del tema de la consciencia; por ejemplo, a través de la educación del individuo humano de "carne y hueso", como han propuesto las diversas críticas al pensamiento habermasiano; pero, antes, habiendo sorteado el análisis de las demás dimensiones de la vida social del ser humano, especialmente el papel de la identidad (Sáez, 2005) dentro de la modernidad, y desde una necesaria perspectiva crítica de la misma (Lévinas, 2008).

\section{Supuestos diagnósticos de partida}

Hoy en día, se presenta un evidente agotamiento de las teorías tradicionales del sujeto y, especialmente, de aquellas que se orientaban, hasta hace poco, en función de la denominada filosofía de la consciencia dentro de las ciencias sociales, las humanidades y la misma filosofía social. 
Aunque dicho fenómeno no es nuevo, se manifiesta (finales del siglo $\mathrm{XX}$ y primeras dos décadas del siglo XXI) en un marco en que las ciencias sociales y humanas en general se han expandido y manifiestan múltiples formas de descripción y explicación del fenómeno social humano, pero mediante teorías opuestas en muchos casos y, en otros, de naturaleza parcial o parcelaria, sin una evidente concatenación metatéorica. O bien, como en el caso de Niklas Luhmann (2007), recurriendo a una teoría general, donde la vida humana tiende a quedar por fuera, es decir, muy lejos de una teoría crítica propuesta, por ejemplo, por autores como Enrique Dussel o Boaventura de Sousa Santos, para quienes la vida humana no puede desligarse de las funciones cognitivo/sociales. Estas últimas son, precisamente, el quid de lo social, desde el punto de vista de una fundamentación ética de naturaleza volitiva (promesa evidente de la Ilustración moderna).

Dicho agotamiento causa diversos problemas en la comprensión general de las ciencias sociales, el cual se manifiesta en los procesos de investigación y en un cierto carácter plano sencillamente sumativo del conocimiento que se produce, día a día, sin alcanzar principios generales que la guíen, a diferencia de las ciencias de la naturaleza, últimas que tienden a una cierta univocidad paradigmática y, por ende, a explicaciones globales válidas sobre su objeto.

Este agotamiento, además, desborda las teorías tradicionales, al no poder dar cuenta de fenómenos claramente novedosos de la realidad social, ya tratados por diversos teóricos, entre los cuales se destaca:

- La separación entre la acción y los criterios de justificación de la acción u orientación de la acción en todos los planos de la actividad humana

- La renuencia de la realidad social a responder o atarse solamente a una teoría del denominado sujeto individual.

- La aparente desvinculación entre las acciones individuales y los ideales de justificación de la misma, los cuales producen una heterogeneidad de fenómenos adicionales (globalización no crítica de criterios semánticos, aparente irracionalidad de la acción humana, por ejemplo, hacia la vida y hacia la naturaleza; irrelevancia de la racionalidad en términos puros de orientación y legitimidad ontológica de la acción; crisis de las orientaciones políticas de las acciones transformativas; desarraigo de la condición humana bajo sistemas técnico-sociales profundamente determinantes de la acción, etcétera).

- La evidente complejización de la actividad y el mundo humanos a nivel 
planetario, con efectos locales y regionales específicos dentro de la noosfera

- La aparente impredecibilidad de los denominados sistemas sociales (Parsons, 1984; Rodríguez y Arnold, 1999) o humanos, sin contar con el desarrollo de visiones pesimistas sobre el futuro, aunque no del todo fundamentadas (esto sucede sobre todo en el marco de la producción cultural de los países centrales o vanguardias del capitalismo).

\section{Conceptos generales necesarios}

Este contexto de desfase entre la acción y la naturaleza social del ser humano requiere conceptos precisos que permitan dar cuenta de los alcances de los fenómenos enunciados anteriormente, superando el voluntarismo, las viejas teorías gregarias decimonónicas (o meramente etológicas), las figuras de la sociedad y la asociación (ya evidentemente agotadas), el comunitarismo neofascista, la vieja concepción de la división social del trabajo en términos económicos, sociales y políticos, los anclajes éticos fundados exclusivamente en las teorías sociológicas y el activismo social voluntarista y sin fundamento efectivamente consciente, etcétera.

Igualmente, se requiere conceptos simples no derivados (ni emulativos) de las ciencias naturales (como se ha planteado, no hace falta decirlo, en muchas ocasiones), que respondan a la realidad social compleja actual y a sus derroteros históricos.

Sin duda, las categorías de acción y sentido de la acción (en la tradición sociológica y filosófica weberiana) siguen teniendo vigencia, siempre y cuando se las desvincule (aunque no completamente, como se verá) selectiva y justificadamente de la filosofía o la metateoría de la consciencia (Searle, 2009) o el denominado entendimiento (Chomsky, 1973).

Las categorías o conceptos pueden enmarcarse dentro de la teoría del lenguaje y la comunicación, pero también en el marco de los conceptos de vida (Marx, Habermas y, en general, la tradición del pensamiento crítico), complejidad (Morin, Maturana y otros autores) y sistema (principalmente, Habermas y Luhmann).

Por ahora, entre los conceptos centrales requeridos apunto a algunos usualmente utilizados, pero resemantizados, así como a otros de carácter 
neológico que, a mi criterio, expresan realidades tangibles dentro del mundo social actual. Dichas categorías pueden enumerarse de la siguiente forma:

- Inductores semánticos o unidades mínimas cargadas de sentido parcial o totalmente dentro de procesos de comunicación (semántica social). Sobre la definición clásica de semántica ver Guiraud, 1982

- Acción social, ya señalada

- $\quad$ Sentido de la acción, ya señalado también (este proviene de la tradición weberiana: Weber, 2012).

- Eventos pragmáticos o unidades finales de la acción mediadas por al menos un inductor semántico (pragmática social)

- Actantes, como concepto general que supere (aunque englobándolo) el viejo concepto de "sujeto" y su actual y aparente "pérdida" (Lagorio, 1998).

- $\quad$ Sistema

- Vida (y mundo de la vida)

- Colonización del mundo de la vida. En sentido categorial, esta idea habermasiana implica la división de los movimientos reales en tres formas: sistema, zonas neocolonizadas del mundo de la vida en los dos primeros casos es donde encontramos las inducciones de bajo contenido cultural y cognitivo y mundo de la vida propiamente dicha. Para este último caso véase la siguiente tabla:

\section{Tabla 1. Ámbitos pragmáticos según la orientación de la acción}

\begin{tabular}{|l|l|}
\hline \multirow{2}{*}{ En relación con el sistema } & $\begin{array}{l}\text { Tipo 1: Sistémicos en sentido estricto (que no proceden } \\
\text { de una inducción semántica, aunque pueda ser semiótica } \\
\text { o simplemente lingüística en general). }\end{array}$ \\
\cline { 2 - 2 } & $\begin{array}{l}\text { Tipo 2: Parasistémicos (que implican tanto una col- } \\
\text { onización de la vida, como una inducción semántica } \\
\text { mínima imbuida dentro de tal colonización). }\end{array}$ \\
\hline $\begin{array}{l}\text { En relación con el mundo de } \\
\text { la vida }\end{array}$ & $\begin{array}{l}\text { Tipo 3: Propios o estrictos -culturales- del mundo de la } \\
\text { vida }\end{array}$ \\
\hline
\end{tabular}

Fuente: Habermas (2007a y 2007b)

Tómese en cuenta que no trabajaremos los inductores no semánticos (del tipo 1), ya que estos pertenecen, sin duda, a una teoría mucho más general del sentido que no es posible desarrollar acá, misma que demanda la descripción con mayor amplitud de la relación entre cuerpo, mente y acción. Expondré de forma breve, por lo tanto, algunos elementos descriptivos sobre 
estas categorías, sin dejar de expresar que, en otro momento, tendré que referirme a ellas con mayor alcance y profundidad. Por lo que el presente documento debe entenderse como una introducción general al problema señalado de la relación sentido-acción social volitiva y no volitiva.

\section{Inductores semánticos y eventos pragmáticos: el sistema semántico-pragmático}

Se define como inductor semántico a la unidad mínima semántica, es decir, capaz de ser portadora de un sentido, de un significado, de manera activa, que puede manifestarse como una acción de naturaleza social (son inductores semánticos, pues, los establecidos por la lingüística como signos: índices, íconos y símbolos; más aquellos complejos que resultan de la unión de estas tres formas de significación; y, como acabo de decir, otros de naturaleza no semántica). Fuera de esta naturaleza solo existen aproximaciones no semánticas -aunque sí, probablemente, semióticas-, por lo que podemos decir que los inductores semánticos se originan dentro del mundo humano. Salvo inductores emocionales, volitivos o perceptuales...insisto que no nos ocuparemos aquí de estas últimas tres categorías, aunque sí de los efectos pragmáticos de la misma naturaleza.

De igual manera, se denomina evento pragmático a cualquier efecto orientado por uno o varios inductores semánticos derivados de procesos comunicativos en su sentido más simple de intercambio en una o doble vía con efectos de retroacción (respuesta de un polo de las relaciones comunicativas actanciales y de los trozos comunicantes -mecánicos- del sistema). Siguiendo la semántica estructuralista (aunque no exclusivamente ella, sino también la lingüística cognitiva contemporánea, ligada, a su vez, a la ya aludida indirectamente semántica cognitiva), podemos enumerar los inductores semánticos mediante el criterio de su función social; es decir, se denomina como evento pragmático al conjunto de efectos prácticos, sean dentro de un marco consciente o inconsciente de la acción social, que implican la participación de por lo menos los siguientes factores:

- Uno o varios inductores semánticos

- Uno o varios actantes 
- Un evento pragmático o efecto práctico dentro de la realidad humana, no exclusivamente comunicativo, es decir, positivo en sentido estricto

- Un enlace de sentido mínimo, que conecte la relación condicionante inductor semántico-evento pragmático.

Podemos poner un par de ejemplos dependiendo del carácter simple, medianamente complejo o altamente complejo. En futuros trabajos esperamos contar con una clasificación teóricamente más coherente y constructivamente compleja de dichos inductores. Los eventos pragmáticos pueden dividirse, por ahora, en simples, medianamente complejos y altamente complejos en relación con su grado de alcance respecto de la condición cultural y cognitiva humana; pero, también, sistémica, propia de los inductores semánticos que los inducen.

Un inductor semántico simple es aquel que corresponde a un bajo grado de cognitividad y sentido cultural, como un nmótico de X (un inductor/ proceso semántico ligado a la decantación o inducción nmemótica de un dato positivo - es decir, constatable por medio de la ciencia-cualquiera $X$ ) o un cursor de $Z$ (un indicador u orientador unidimensional, bi, tri o multidimensional que señala el curso posible de la acción o del movimiento de una actancia cualquiera, sea en el espacio real, simbólico/comunicativo o imaginario; Mora, 2015).

En el segundo caso nos encontramos con un grado medio de cognitividad y contenido cultural, las ideas (idea de X) y las normas (norma de Z). En este caso se requiere una mayor cognición para que el evento pragmático X o $Z$ pueda darse.

Por ello, señalé al principio que, aunque las ideas expuestas en este ensayo tienen como finalidad trascender la teoría de la consciencia, como a priori teórico, no por ello suponen una negación de la misma en pos del principio de la vida. Ya que, fuera de la consciencia, dentro de cualquier teoría de la vida humana, la negación de la misma se reduce a un sinsentido ontológico (lo cual no implica que la consciencia explique completamente, como en tiempos históricos anteriores, la estructura de lo real y de la vida intramundanamente entendida). Se trata de eventos pragmáticos complejos que, desde el punto de vista social, suponen ricos contenidos pragmáticos y que solo pueden ejecutarse mediante la participación de actantes tipo 
sujeto, de forma volitivamente guiada y mediante la mediación de la consciencia. En este tercer caso se puede poner el ejemplo de una teoría de X, o una cosmogonía Z.

De acuerdo con esto se deriva que los inductores semánticos, en su relación práctica y en su decantamiento (como eventos pragmáticos), no pueden desligarse de, por lo menos, las siguientes teorías:

- La teoría del lenguaje ya dicha.

- Las teorías cognitivas (e, incluso, pese a las críticas de las que han sido objeto, de la mente -McDowell, 2003- y la consciencia).

- Las teorías contemporáneas de la acción y del acto en sentido estricto (epistemológicamente positivo). (Nota: Me refiero a una teoría puramente positivista, misma que he intentado aplicar al presente tema y en el proceso de la elaboración de este texto, sin resultados satisfactorios o al menos primariamente válidos desde un punto de vista metateórico.)

- La presencia del sistema

- La naturaleza y constitución fenomenológica del concepto de mundo de la vida

- Una hermenéutica interpretativa o traductora que ligue evento pragmático e inductor semántico, bajo la figura de una necesaria actancia.

Por ello, señalé al principio que, aunque las ideas expuestas en este ensayo tienen como finalidad trascender la teoría de la consciencia, como a priori teórico, no por ello suponen una negación de la misma en pos del principio de la vida. Ya que, fuera de la consciencia, dentro de cualquier teoría de la vida humana, la negación de la misma se reduce a un sinsentido ontológico. Sin el concepto de consciencia o de mente consciente, todo el aparataje teórico de las ciencias sociales y de la filosofía se desbarata, no permitiendo ninguna explicación de lo real, ni siquiera en sentido fenomenológico.

El acto/sentido/mundo (acto, entonces, que es configurado, en primera instancia, por los "diseños" pragmáticos intramundanos que sobrevienen, a su vez, de una inducción semántica); más los puramente mecánicos del sistema social. La inducción semántica define, pues, el grado de razonabilidad -y no solo racionalidad- de, por ejemplo, la acción instrumental de configuraciones dentro del sistema o dentro del mismo mundo de la vida (por ahora sectores de este). Dicho de otra manera, no hay mundo sin que se le delimite pragmáticamente por medio, al menos, de una inducción de 
un acto en el mundo, es decir, que defina un "diseño pragmático" (un estado sectorial específico del mundo, sea o no razonable).

Al final del presente texto se expondrá, como ya señalé arriba, un primer acercamiento a un glosario descriptivo de los términos utilizados en este texto, de forma que lo hasta ahora dicho pueda aclararse un poco más, y dada la necesidad de resumir las tesis centrales del texto dentro de un espacio de tamaño convencional propio de un artículo.

\section{Actantes}

Se denomina como actantes a las unidades ejecutoras dimensionables de, por lo menos, un inductor semántico en el marco de la realidad social; que supone, a su vez, la operación o realización de por lo menos un evento pragmático positivamente entendido. Con el término actante se busca sortear el problema conceptual referido al sujeto, aunque comprendiéndolo (incluso, en un sentido trascendental) como se verá más adelante; dada la incapacidad de la dupla sujeto/consciencia a la hora de explicar la acción social de forma satisfactoria.

Los actantes pueden clasificarse de acuerdo a la relación establecida entre su grado de relación con los procesos de orden biológico referidos a la vida orgánica (alto, medio y bajo -aquí remito a algunas propuestas que ven el "uso" histórico de condiciones "postorgánicas"-) y su alcance pragmático/ cognitivo (altamente complejo, medianamente complejo y simple, según lo visto atrás). Cosa que podemos representar de la siguiente forma (hay que recalcar que la posición de dichos actantes no es estática, sino que se está moviendo en el doble sentido izquierda-derecha y arriba-abajo, dependiendo de las circunstancias históricas; así como que el carácter cognitivo es una variable necesaria en la evaluación de la naturaleza de los actantes, según lo que propongo en este texto): 


\section{Tabla 2. Tipo de actancias según carácter biológico y pragmático/cognitivo de la acción}

\begin{tabular}{|c|c|c|c|}
\hline \multirow{2}{*}{$\begin{array}{c}\text { Tipo de actancia } \\
\text { según carácter } \\
\text { biológico }\end{array}$} & \multicolumn{2}{|c|}{ Tipo de actancia según alcance pragmático/cognitivo } \\
\cline { 2 - 4 } & $\begin{array}{c}\text { Simple } \\
\text { (Instructores) }\end{array}$ & $\begin{array}{c}\text { Medianamente } \\
\text { complejo (Operadores) }\end{array}$ & $\begin{array}{c}\text { Altamente complejo } \\
\text { (Agentes) }\end{array}$ \\
\hline Biológico & Protobioides & (Clones cognitivos) & Sujetos \\
\hline Semibiológico & Servoinstructores & Bioinstructores & Avatares \\
\hline Interbiológico & (Simbiontes) & Simbioides & (Biorroides) \\
\hline No biológico & Servomecanismos & Robots & Sistemas expertos/(IA) \\
\hline
\end{tabular}

Fuente: Habermas (2007a, pp. 365-366) y Gallo (2000)

La idea de esta división y de la anterior es reconocer, a través de ellas, la complejidad de la acción social, más allá de lo propuesto por las teorías estrictas del individualismo metodológico y la teoría marxista de la "consciencia de clase" (es decir, del llamado "sujeto histórico"), es decir:

- Que ha habido una multiplicación histórica tanto de los eventos pragmáticos como de las actancias.

- Que, hasta cierto punto, la existencia del sujeto (tal y como lo entienden el liberalismo clásico y el marxismo -categoría de sujeto histórico-) es minoritaria o tiende a ser minoritaria.

- Que una teoría social no debiese desconocer este revés o agotamiento de la teoría clásica de la acción.

- Que en muchas de las dimensiones en que, como actantes, valga la redundancia, actuamos, lo hacemos de forma mecánica en función de los inductores semánticos, es decir, que actuamos (mediante eventos semántico-pragmáticos prefabricados) a espaldas de cualquier modalidad de la consciencia, como plantean las teorías sociales clásicas y pese a la naturaleza semántica de los inductores señalados.

- Que la cantidad de actancias se ha multiplicado, y que estas responden al sistema y no tanto a la vida (este planteamiento se desprende de la teoría habermasiana). No obstante, que las ciencias sociales siguen pensando, en la mayoría de los casos, bajo el binomio "individuo/sociedad", es decir, bajo premisas sicologistas y sociologistas.

- Que, en el caso humano, la teoría de la cultura supera con creces el denominado proceso de socialización, siendo sustituido este por el "movimiento" 
de inductores semánticos de carácter simple, que operan más allá de las individualidades humanas o a través de ellas, pero de forma indirecta (en este caso, sí se dan procesos de socialización dentro del marco del sistema y fuera del circuito de la vida -"mundo de la vida"-).

\section{Conclusión: vida y sistema}

La división analítica entre vida y sistema proviene de la teoría habermasiana y permite analizar una compleja relación entre el proceso de historización culturizada humana (mundo de la vida), la independización creciente (sobre todo dentro de la modernidad) del sistema y el concepto agregado de parasistema (sector colonizado o en vías de colonización del mundo de la vida). Aquí planteo, como parte de influencia del sistema y el parasistema, el papel de las actancias (y las relaciones que se producen a partir de ellas, es decir, las relaciones sociales); concepto, este último, necesario para poder abarcar formas de acción individualizada de bajo contenido cognitivo o de contenido cognitivo disgregado en diversas actancias de las ya señaladas; es decir, que superan el concepto tradicional de "individuo" del sentido común y de las ciencias sociales, así como los conceptos de acción y relación sociales, utilizados en las ciencias humanas.

Habermas (2007a y 2007b) únicamente se preocupa por la explicación de dos de los elementos que denomina sistema, a saber, el dinero y el poder. Sin embargo, consideramos que el sistema es mucho más amplio y que elementos que Habermas ubica en el mundo de la vida, pertenecen ya, sin duda, al sistema, al aparecer diversas manifestaciones actanciales, no existentes, históricamente, hasta ahora. Mientras que otro autor, Luhmann (2007), abandona por completo una teoría del sujeto a favor de la teoría de sistemas y subsistemas y de la comunicación acaecida dentro de/entre subsistemas sociales mediados por "entornos".

Tomando como referencia lo hasta aquí dicho, podemos caracterizar las formaciones sociales contemporáneas de la siguiente forma (la enumeración a realizar no pretende agotar en ningún sentido el tema en cuestión):

- La vida (Capra, 2011; Mosterín, 2009; González, 2009) o "mundo de la vida" cubre dentro de la modernidad pocas facetas de la actividad humana, ya que: $\checkmark \quad$ El sistema ha invadido (en el decir de Habermas: "ha colonizado") gran 
parte de la vida, por un lado mediante la limitación de las agencias; y, por otro, mediante el surgimiento de múltiples instructores (rutas de acción prefabricadas dentro del sistema, es decir: protobioides, simbioides, servomecanismos y servoinstructores); operadores (bioinstructores, robots); e, incluso, avatares y sistemas expertos; es decir, por el incremento cualitativo y cuantitativo de formas artificiales (no biológicas o semibiológicas) de actancia; independientemente de la mediación del cuerpo humano, partes de él o de prótesis y ampliaciones metaprotésicas, reales o virtuales del mismo.

- En general, las formaciones sociales modernas son procesos de ordenamiento artificial de la actividad humana. Este ordenamiento responde a los aspectos ya señalados atrás y según los componentes que se describirán enseguida como parte del glosario.

- Hasta el momento, las humanidades y las ciencias sociales en específico no se han pronunciado, suficientemente, sobre esta condición de la modernidad, ni sobre los efectos de la misma en la vida cotidiana y práctica de los seres humanos (Sibila, 2005). Dejando por la libre (o contribuyendo con ellas) las modalidades complejas de socialización por medio exclusivo o semiexclusivo de la técnica, sin que medie una reflexión efectivamente crítica y tecnológica (es decir, desde una "ciencia de la técnica"-techné + logos-).

\section{Glosario básico}

El siguiente glosario solo cubre conceptos y definiciones no expresados o definidos de manera directa en el cuerpo del texto principal. Otros términos pueden deducirse de lo expresado anteriormente.

1. (Clon): Copia biológica de un ser viviente o de una parte (órgano) del mismo. Se pone entre paréntesis porque en teoría la investigación clónica se ha detenido en parte, sin llegar a la clonación humana, pero sí de partes orgánicas a través de técnicas transgénicas (para la producción médica de órganos humanos para su posterior trasplante).

2. (Clon cognitivo): Ver definición anterior

3. (IA): Inteligencia artificial. Aparece entre paréntesis, pues se ha llegado poco más allá de los sistemas expertos en términos estrictos del sentido del término inteligencia. Cf.: González, 2010, pp. 153-176; Capra, 2011.

4. (Simbiontes): Máquinas con componentes biológicos de origen no humano o humano. Este es el caso de los biocircuitos. 
5. Acción social: Toda acción (evento pragmático) que realiza un actante y que supone una intervención en el mundo, a partir de la orientación del sistema, del mundo de la vida o de ambos.

6. Alcance cognitivo: Grado en que un actante logra determinada capacidad cognitiva. Aunque resulta posible medir el grado y sus cualidades, aquí solamente se ha apreciado el grado en función de tres niveles: bajo, medio y alto.

7. Bioinstructores: Instructores de carácter biológico que operan como facilitadores de actividades mecánicas o biológicas. Este es el caso de la investigación sobre nanobots de uso médico, que, momentáneamente, establecen simbiosis con organismos humanos o no humanos.

8. Biorroides: Ser humano al que se le ha cambiado gran parte de su cuerpo por aditamentos tecnoartificiales. Se incluye aquí la unidad hombre-sistema experto para usos industriales, espaciales o militares.

9. Cosmogonía: Visión compleja de un mundo que contempla la descripción y la posición de un determinado conjunto de actantes en este mundo.

10. Cultura: Producción histórica, sea sistémica o vital, que condiciona la acción de los actantes.

11. Emoción: Conjunto de inductores semióticos y valorativos que se refieren a algo en el mundo, de forma no semántica, aunque sí pragmática.

12. Idea: Unidad cognitiva que refiere a una descripción o explicación de algo en el mundo.

13. Mente: Unidad cognitiva compleja que tiene por característica principal el acceso a su propia condición como mente, es decir, que es capaz de un mínimo de consciencia.

14. Mundo: Unidad vivencial habitada o construida por uno o más individuos

15. Nmóticos: Inductores semánticos y eventos pragmáticos que localizan inductores semánticos "dentro" de la memoria de un actante.

16. Norma: Directriz que indica un comportamiento necesario o la prohibición de una acción cualquiera.

17. Operadores: Actancias caracterizadas por una mediación cognitiva de medio alcance

18. Protobioides: Mecanismos constituidos por componentes biológicos que funcionan como unidad igualmente mecánica y no tanto biológica. Podríamos denominarlos también máquinas protobiológicas.

19. Robot: Actante artificial (virtual o mecánico) que "actúa" mediante un programa operativo que "le permite" emular o llevar a cabo determinadas funciones.

20. Sentido de la acción: Inductor semántico garante de que una específica acción de un actante no devenga como meramente aleatoria. El sentido supone un ordenamiento o un cambio consciente o no consciente del ordenamiento 
social en un momento determinado (ver definición en Webber, 2012, 6ss; en el sentido de una acción de un individuo que refiere a otro individuo).

21. Signo: Unidad semántica de un significante y un significado. Incluye índices, íconos y símbolos.

22. Simbioide: Unión entre una función o controlador biológico de mediana capacidad cognitiva y una terminal mecánica

23. Sistema experto: Producto informacional con altas capacidades de procesamiento y análisis de la información, previo al desarrollo de una I. A. Ver Kendall y Kendall, 1995, p. 3

24. Sujetos: Actancias caracterizadas por un elevado alcance pragmático-cognitivo

25. Teoría: Forma específica de describir el mundo mediante un conjunto concatenado de leyes y paradigmas

26. Volición: Validación de determinado objeto en el marco de una determinada escala no semántica, ligada con la voluntad. Acto en función de esta última

\section{Referencias}

Blumenberg, H. (2013). Teoría del mundo de la vida. Buenos Aires: Fondo de Cultura Económica.

Capra, F. (2011). La trama de la vida. Una perspectiva de los sistemas vivos (6 ed.). Barcelona: Anagrama.

Chomsky, N. (1973). El lenguaje y el entendimiento. Barcelona: Seix Barral.

Díaz, J. L. (2008). La conciencia viviente. México: Fondo de Cultura Económica.

González, J. (2009). Ontología y ciencias de la vida. En J., González (coord.), Filosofía y ciencias de la vida. México: Fondo de Cultura Económica. (Pp. 123-133).

González, J. (2010). La teoría de la mente: de la inteligencia artificial a la inteligencia híbrida. En: Diosdado, C., Rodríguez, F., Arana, J., Neurofilosofía: perspectivas contemporáneas. México: Plaza y Valdéz. (Pp. 153-176).

Gallo, R. (2000). Diccionario de la ciencia y la tecnología. Universidad de Guadalajara, México.

Guiraud, P. (1982). La semántica (2ª ed.). México: Fondo de Cultura Económica.

Habermas, J. (2007a). Teoría de la acción comunicativa I. México: Taurus.

Habermas, J. (2007b). Teoría de la acción comunicativa II. México: Taurus.

Kendall, K. y Kendall, J. (1995). Análisis y diseño de sistemas (3ª ed.). México: Prentice Hall Hispanoamericana.

Lagorio, C. (1998). Cultura sin sujeto. El dominio de la imagen en la posmodernidad. Buenos Aires: Biblos.

Lévinas, E. (2008). Nombres propios. Salamanca: KADMOS.

Luhmann, N. (2007). La sociedad de la sociedad. México: Herder.

Marx, K. (1999). La ideología alemana. El Manifiesto Comunista. El papel del trabajo en la transformación del mono en hombre. Costa Rica: Alma Mater.

McDowell, J. (2003). Mente y mundo. Salamanca: Ediciones Sígueme. 
Mora, M. (2015). Algunos problemas de fundamentación y justificación sociológica: apuntes en el marco del debate teórico de la sociología contemporánea. Revista ABRA. Volumen 35, N. 50. Heredia, Costa Rica: EUNA .(Pp. 1-19).

Mosterín, J. (2009). ¿Qué es la vida? En J., González (coord.), Filosofía y ciencias de la vida. México: Fondo de Cultura Económica. (Pp.33-159).

Parsons, T. (1984). El sistema social (2ª ed.). Madrid: Alianza Editorial.

Rodríguez, D. y Arnold, M. (1999). Sociedad y teoría de sistemas (3ª ed.). Santiago de Chile: Editorial Universitaria. (Pp. 33-59)

Sáez, M. (2005). Charles Taylor: identidad personal y moral. En M., Rodríguez (coord.), La mente en sus máscaras. Ensayos de filosofía de la psicología. Madrid: Biblioteca Nueva. (Pp. 101-121).

Sanfélix, V. (2003). Mente y conocimiento. Madrid: Biblioteca Nueva.

Searle, J. (2009). La conciencia. En J., González (coord.), Filosofía y ciencias de la vida. México: Fondo de Cultura Económica. (Pp. 60-93).

Serres, M. (2011). Variaciones sobre el cuerpo. México: Fondo de Cultura Económica.

Weber, M. (2012). Economía y sociedad. México: Fondo de Cultura Económica. 